\title{
Caracterização das atividades avançadas de vida diária (AAVDS): um estudo de revisão
}

\section{Characterization of advanced activities of daily living (AADL): a review}

\author{
Eliane Golfieri Dias ${ }^{1}$, Yeda Aparecida de Oliveira Duarte², Maria \\ Helena Morgani de Almeida ${ }^{3}$, Maria Lúcia Lebrão ${ }^{4}$
}

DIAS, E. G.; DUARTE, Y. A. O.; ALMEIDA, M. H. M.; LEBRÃO, M. L. Caracterização das atividades avançadas de vida diária (AAVDS): um estudo de revisão. Rev. Ter. Ocup. Univ. São Paulo, v. 22, n. 1, p. 45-51, jan./abr. 2011.

RESUMO: Introdução: As atividades avançadas de vida diária (AAVDs) envolvem atividades cotidianas mais complexas do que as atividades básicas e instrumentais de vida diária, as quais não são incluídas de forma sistemática na avaliação funcional do idoso. Considera-se a necessidade de melhor definição e difusão do termo, a partir da caracterização das atividades incluídas no conjunto das AAVDs. Objetivo: Caracterizar os estudos sobre AAVDs em periódicos científicos nacionais e internacionais quanto aos conceitos e definições utilizados, grupos de atividades e considerações sobre o processo de avaliação. Material e método: Estudo de revisão envolvendo as bases de dados Medline, Scielo, Lilacs, Pubmed, Journal Storage, MUSE (1984-2008) e busca manual. Resultados: A partir de 212 resumos selecionados, identificou-se 35 estudos que utilizaram o termo AAVDs. De forma geral, os estudos basearam se no trabalho de Reuben e col (1989). Os resultados reforçam características de complexidade, subjetividade e influência direta de padrões socioculturais locais, melhores condições de funcionalidade e qualidade de vida no desempenho das AAVDs. Foram identificados como domínios inter-relacionados de atividades: social, físico, produtivo e de lazer. Conclusão: Defende-se o uso do termo AAVDs e incorporação destas à avaliação gerontológica, bem como em programas de promoção à saúde e reabilitação.

DESCRITORES: Atividades cotidianas; Avaliação geriátrica; Atividades de lazer; Participação social; Idoso.

1. Terapeuta Ocupacional. Mestre e doutoranda em Saúde Pública - Faculdade de Saúde Pública - USP.

2. Professora Livre Docente do Departamento de Enfermagem Cirúrgica da Escola de Enfermagem - USP.

3. Professora Doutora do Departamento de Fisioterapia, Fonoaudiologia e Terapia Ocupacional da Universidade de São Paulo.

4. Professora Titular do Departamento de Epidemiologia da Faculdade de Saúde Pública - USP. E-mail: mllebr@usp.br

Endereço para correspondência: Eliane Golfieri Dias. Rua Salvador Simões, 345 - Ipiranga. São Paulo. CEP: 04276-000. e-mail: egolfieri@usp.br 


\section{INTRODUÇÃO}

discussão inicial sobre a incorporação de
atividades mais complexas na avaliação fun-
cional do idoso além das atividades básicas (ABVDs) e instrumentais (AIVDs) iniciou-se com Rubenstein et al. (1988). Esses autores destacaram a necessidade da inclusão de atividades relacionadas ao desempenho de papéis sociais, com avaliação do funcionamento físico, mental e social dos idosos.

Reuben e Solomon (1989) propuseram o uso dos três níveis funcionais de atividade: básicas (ABVDs), intermediárias (AIVDs) e avançadas (AAVDs). AAVDs foram inicialmente definidas como atividades voluntárias, específicas para cada indivíduo e influenciadas por fatores socioculturais e motivacionais. Os autores defenderam a necessidade de aprofundamento no estudo destas atividades, sugerindo sua correlação com a qualidade de vida e saúde mental dos idosos, além de dificuldades nas AAVDs serem preditoras de declínio funcional progressivo em fase inicial.

As atividades sociais, produtivas e de lazer ganharam destaque nos últimos anos, sendo incluídas nas políticas públicas como estratégias de programas de promoção à saúde, especialmente as atividades sociais e de lazer. Há referência da importância da promoção destas atividades nas políticas de saúde, como a Política Nacional de Saúde do Idoso (BRASIL, 2003) e Política Nacional de Promoção à Saúde (BRASIL, 2006), como também no Estatuto do Idoso (BRASIL, 2003):

“Art. 3o É obrigação da família, da comunidade, da sociedade e do Poder Público assegurar ao idoso, com absoluta prioridade, a efetivação do direito à vida, à saúde, à alimentação, à educação, à cultura, ao esporte, ao lazer, ao trabalho, à cidadania, à liberdade, à dignidade, ao respeito e à convivência familiar e comunitária" (ESTATUTO DO IDOSO, 2003).

Na prática clínica, a avaliação das atividades avançadas de vida diária não é realizada de forma sistemática e não foi incorporada à avaliação gerontológica. Poucos autores têm se dedicado ao estudo das AAVDs na avaliação funcional do idoso, apesar do interesse crescente pela realização de pesquisas sobre a importância da participação da pessoa idosa em atividades sociais, produtivas e de lazer na melhoria de suas condições de saúde e de qualidade de vida.

Considerando-se a necessidade de melhor definição e difusão do termo, da caracterização das atividades incluídas no conjunto das AAVDs, este artigo apresenta uma revisão bibliográfica sobre o tema.

\section{OBJETIVO}

Caracterizar os estudos sobre AAVDs em periódicos científicos nacionais e internacionais em relação aos conceitos e definições utilizados.

\section{MATERIAL E MÉTODO}

Foram pesquisadas as seguintes bases de dados dos campos da saúde e das ciências humanas: Medline, Lilacs, Pubmed, Journal Storage, MUSE, Scielo no período de 1984-2008. A busca foi realizada com descritores e palavras, em decorrência do reduzido número de referências localizadas sobre AAVDs. Foram utilizados os termos atividade(s) avançada(s) de vida diária, atividades sociais e idoso, atividades produtivas e idoso, atividades de lazer e idoso, avaliação geriátrica. A escolha da data inicial deve-se à década de publicação de Rubenstein et al. (1988), considerado como o primeiro estudo que mencionou a importância da incorporação das atividades de maior complexidade na avaliação geriátrica abrangente. Não foi possível realizar uma busca padronizada, com o uso dos mesmos descritores e palavras nas diferentes bases de dados, devido às divergências dos descritores que são utilizados nas bases de dados nos campos das ciências humanas e saúde e pelos próprios autores em seus estudos. Outra estratégia utilizada foi a busca manual em listas das referências utilizadas nos artigos selecionados e em bases de dados das principais universidades brasileiras.

\section{RESULTADOS E DISCUSSÃO}

Foram selecionados 212 resumos correspondentes às atividades sociais, físicas, produtivas e de lazer no envelhecimento. Destes, foram selecionados 35 estudos que utilizaram a terminologia AAVDs. A partir dos resultados encontrados foram exploradas as características das AAVDs, grupos de atividades envolvidas e considerações sobre a avaliação destas atividades.

De modo geral os estudos utilizaram Reuben et al. (1990) como referência para o conceito de AAVDs. Em sua maioria os estudos referiam-se às AAVDs como componentes da avaliação funcional do idoso.

\section{CARACTERÍSTICAS DAS AAVDS}

\section{As AAVDs são as atividades cotidianas de maior complexidade}

As AAVDs foram citadas em quinze estudos como atividades mais complexas do que as ABVDs e AIVDs, 
porém não diretamente relacionadas à sobrevivência direta na comunidade. As AAVDs são relacionadas aos mais altos níveis de funcionalidade. Os autores justificam que estas atividades envolvem os mais altos níveis de aprendizagem e são influenciadas por habilidades físicas, cognitivas, emocionais e sociais mais complexas. (ALARCÓN, 1996; ALTIMEI LOSADA et al., 2007; BORGES, 2006; CALDAS, 2003; FONSECA; RIZZOTTO, 2008; GÓMEZ PORTILLA et al., 2008; MANUBENS BERTRAN, 2003; MARTOS MARTÍN, 2005; MINCATO; FREITAS, 2007; PASCHOAL, 2002; PENNING, 2002; REUBEN et al., 1990; REUBEN; SOLOMON, 1989; ROLIM; FORTI, 2005; ROMERO; MORUNO, 2003; RUBENSTEIN et al., 1990; SILVA, 2005). sociais

\section{As AAVDS relacionam-se à execução de papéis}

Doze estudos referiram que as AAVDs estão relacionadas à execução de papéis sociais, favorecendo o desenvolvimento pessoal, integração e habilidades sociais (ALARCÓN, 1996; CALDAS, 2003; FONSECA; RIZZOTTO, 2008; JAVIER GÓMEZ, 2003; LOZANO, 2004; MANUBENS BERTRAN, 2003; MARTOS MARTÍN, 2005; MINCATO; FREITAS, 2007; PAIER, 1994; POLÔNIO LÓPEZ, 2002; ROMERO; MORUNO, 2003; SALVADORES, 2003).

\section{O desempenho das AAVDs envolve independência no controle dos ambientes físico e social}

O engajamento nas AAVDs foi associado em quatro estudos como fator de independência no controle do ambiente físico e social e cumprimento dos papéis sociais em diferentes contextos, tais como lazer, trabalho e engajamento social. As AAVDs favorecem o desenvolvimento e exercício das habilidades pessoais e a inclusão social (MANUBENS BERTRAN, 2003; POLÔNIO LÓPEZ, 2002; SALVADORES, 2003; ROMERO; MORUNO, 2003).

As AAVDs foram relacionadas a um melhor estado funcional e qualidade de vida

As AAVDs foram utilizadas como variável independente nos estudos selecionados, sendo relacionadas em seis estudos a altos níveis de capacidade funcional, qualidade de vida e saúde mental no envelhecimento (ALARCÓN, 1996; BORGES, 2006; FONSECA; RIZZOTTO, 2008; ROLIM; FORTI, 2005; POVEDA et al., 2005; ROLIM, 2005; RUBENSTEIN et al. 1990) Alguns autores relacionam as AAVDs também a um melhor funcionamento físico e cognitivo (JOHNSON; WOLINSKY, 1993; MANUBENS BERTRAN, 2003; MELO, 2009).

Apesar da referência do desempenho das AAVDs a melhores condições de saúde, funcionalidade e qualidade de vida não há menção entre os estudos da utilização destas atividades como estratégias para a promoção da saúde. Percebe-se que os estudos concentram-se em visões mais específicas sobre prevenção de agravos e manutenção da funcionalidade.

Comprometimentos nas AAVDs são indicadores precoces da deterioração da capacidade funcional

As AAVDs foram referidas como preditoras de perdas funcionais futuras, de forma que o declínio nestas atividades representa um indicador precoce de redução da capacidade funcional. Seu monitoramento pode detectar mudanças na capacidade funcional ao longo do tempo (FONSECA; RIZZOTTO, 2008; GARMAN; COHEN, 2002; LOBO; PEREIRA, 2007; LOZANO, 2004; MARTOS MARTÍN, 2005; PAIXÃO Jr; REICHENHEIM, 2005; PASCHOAL, 2002; POLÔNIO LÓPEZ, 2002; REUBEN; SOLOMON, 1989; RUBENSTEIN et al., 1990).

A diminuição do desempenho de AAVDs no cotidiano foi relacionada a isolamento social e restrições nas demais atividades cotidianas (BORGES, 2006).

As AAVDs são atividades subjetivas, relacionadas a fatores culturais e motivacionais

Alguns autores destacaram a importância da subjetividade, influências culturais e motivação para a escolha e envolvimento nas AAVDs (SINDACO et al., 2004; REUBEN; SOLOMON, 1989; RUBENSTEIN et al., 1990).

\section{GRUPOS DE ATIVIDADES COMPREENDIDOS NAS AAVDS}

Apesar de aparentemente baseados na mesma definição de Reuben e Solomon (1989) os estudos citados diferiram quanto aos grupos de atividades descritas como AAVDs, bem como em sua abrangência.

De modo geral, os grupos de maior prevalência 
DIAS, E. G. et al. Caracterização das atividades avançadas. Rev. Ter. Ocup. Univ. São Paulo, v. 22, n. 1, p. 45-51, jan./abr. 2011.

foram: atividades sociais, produtivas e de lazer. Observase que foram incluídos, basicamente, os mesmos conjuntos de atividades: sociais, físicas, produtivas e de lazer. Três trabalhos (PENNING, 2002; HO et al., 2002; JOHNSON; WOLINSKY, 1993) incluíram as AIVD na categoria das AAVDs, no entanto, com referência às atividades de maior complexidade da vida diária. Outros dois estudos (PINKUS et al., 1999; POVEDA et al., 2005) incluíram sob o termo AAVDs somente as atividades físicas de alta demanda funcional, relacionadas ao funcionamento da metade superior e metade inferior do corpo humano.

De modo ilustrativo, apresenta-se a distribuição percentual comparativa das atividades estudadas (Tabela 1), distinguindo-se os estudos que utilizaram a terminologia. Observa-se que a distribuição das atividades é bem semelhante, com destaque aos conjuntos de atividades sociais, produtivas e de lazer. Nota-se o número reduzido de estudos que incluíram o voluntariado, as atividades intelectuais e políticas no conjunto das AAVDs.

Tabela 1 - Distribuição (\%) das atividades segundo citações nos estudos. São Paulo, 2009

\begin{tabular}{lcc}
\hline \multicolumn{1}{c}{ Atividades } & AAVDs & \% \\
\hline Sociais & 29 & 34,5 \\
Lazer & 24 & 28,6 \\
Trabalho & 15 & 17,9 \\
Atividade física/esportes & 10 & 11,9 \\
Políticas & 3 & 3,6 \\
Voluntariado & 2 & 2,4 \\
Intelectuais & 2 & 2,4 \\
Mistas & 1 & 1,2 \\
Solitárias & - & - \\
\hline Total & $\mathbf{8 4}$ & $\mathbf{1 0 0 , 0}$ \\
\hline
\end{tabular}

Fonte: Dados da pesquisa

Foram citadas como exemplos de AAVDs entre os estudos envolvidos nesta revisão: atividades de lazer assistir TV, praticar atividades físicas, dirigir automóvel, preparar refeições para amigos, praticar esportes, realizar atividades manuais, praticar jardinagem, ler jornais, livros ou revistas, realizar passatempos, pescar, tocar instrumento musical, viajar, preencher formulários ou realizar atividades intelectuais; atividades sociais: participar de atividades políticas, manter contatos sociais diversos, fazer chamadas telefônicas, freqüentar restaurantes, participar de eventos sociais, participar de grupos sociais ou religiosos, relacionar-se com amigos e familiares, visitar amigos ou parentes; atividades produtivas: prestação de serviços comunitários, trabalhar, exercer voluntariado, praticar atividades religiosas solitárias.

\section{CONSIDERAÇÕES SOBRE A AVALIAÇÃO DAS AAVDS}

Os autores destacaram a influência de fatores subjetivos, picossociais, das condições e estilo de vida adotado sobre o desempenho das AAVDS. Referem que altos níveis de função, tarefas e papéis sociais tendem a ser mais individualizados, influenciando as escolhas e atividades adotadas no cotidiano. Isto implica em alta variabilidade entre os indivíduos, o que dificulta a criação de uma escala universal que possa medir o desempenho das AAVDs (CALDAS, 2003; JARDIM, 2007; LOBO; PEREIRA, 2007; MANUBENS BERTRAN, 2003; MARTOS MARTÍN, 2005; PAIER, 1994; POLÔNIO LÓPEZ, 2002; REUBEN et al., 1990; REUBEN; SOLOMON, 1989;ROLIM; FORTI, 2005; SALVADORES, 2003; SINDACO et al., 2004).

Apesar da menção da inclusão das AAVDs na avaliação geriátrica abrangente, não foram descritos instrumentos validados específicos para a avaliação das AAVDs (GARMAN; COHEN, 2002; DEMERS et al., 2000; PAIXÃO Jr; REICHENHEIM, 2005).

Cabe destacar a importância de se considerar a interrelação entre os domínios de atividades no momento da avaliação. Apesar da classificação arbitrária das atividades adotadas na maioria dos estudos, entende-se que uma mesma AAVD pode pertencer a mais de um domínio de atividades, por envolver múltiplos papéis e habilidades. Esse caráter misto de muitas das AAVDs somente recebeu destaque no trabalho de Martos Martín (2005). O exemplo a seguir busca explicitar o descrito:

“a prática de esportes pode ser considerada lazer para alguns e atividade produtiva para outros; ir à igreja pode ser uma atividade social para muitos e uma atividade produtiva e de lazer para outros...".

\section{CONSIDERAÇÕES FINAIS}

Com base neste estudo de revisão, pôde-se traçar um panorama das AAVDs. Observou-se a existência de número reduzido de estudos sobre AAVDs, com maior utilização do termo entre os estudos brasileiros, no entanto, sem aprofundamentos conceituais.

Com este estudo, afirma-se a importância do desempenho de AAVDs no envelhecimento. Estas 
atividades constituem-se como estratégia importante para estímulo e preservação de múltiplas habilidades intelectuais, físicas e psicossociais, com o favorecimento da manutenção e fortalecimento da rede de apoio, participação social, autopercepção positiva saúde e de qualidade de vida. Por outro lado, a privação destas atividades pode constituir-se como preditor de doenças e isolamento social, fato este que poderá ser melhor avaliado em pesquisas futuras. Dessa forma, as AAVDs constituem- se como importante estratégia para a promoção da saúde no envelhecimento e devem ser contempladas nas políticas públicas direcionadas à população idosa.

Defende-sea difusão do termoAAVD, considerandose os seus domínios inter-relacionados de atividades: lazer, produtivo e social, bem como a incorporação destas atividades na avaliação funcional do idoso, junto às ABVDs e AIVDs. Isto permitirá maior comparabilidade e aprofundamento de estudos sobre estas atividades.

DIAS, E. G.; DUARTE, Y. A. O.; ALMEIDA, M. H. M.; LEBRÃO, M. L. Characterization of advanced activities of daily living (AADL): a review. Rev. Ter. Ocup. Univ. São Paulo, v. 22, n. 1, p. 45-51, jan./abr. 2011.

\begin{abstract}
Introduction: The advanced activities of daily living (AADL) comprehend more complex activities than basic and instrumental activities of daily living, which are systematically bypassed in geriatric assessment. It requires a better definition and description of the idea through the process of the activities considered in the AAVDs concept. Objective: To describe concepts and terms used in national and international scientific studies about the concepts and AADL definitions, groups of activities and considerations about the evaluation process. Methods: A review study involving Medline, SciELO, Lilacs, Pubmed, Journal Storage, MUSE (1984-2008) and self search. Results: From of 212 selected abstracts, has been identified 35 studies that used the term AAVDs. Usually studies based the work of Reuben et al (1989). The results reinforce characteristics of complexity, subjectivity and direct influence from local sociocultural patterns, better functionality and quality of life in the performance of AAVDs. Were identified as inter-related domains: social, productive and leisure activities. Conclusion: Support the use of the term AAVDs and the incorporation of these in geriatric assessment, as well as programs of health promotion and rehabilitation.
\end{abstract}

KEY WORDS: Activities of daily living; geriatric assessment; Leisure activities; Socail participation; Aged.

\title{
REFERÊNCIAS
}

ALARCÓN, T. Valoración geriatrica: utilidad predictiva en el estudio y seguimiento del paciente geriátrico hospitalizado.1996. [Tese - doutorado] Madrid:. Facultad de Medicina de la Universidad Complutense de Madrid, 1996.

BORGES, S.M. Relação entre função visual e capacidade funcional na velhice [Dissertação - Mestrado]. Campinas: Faculdade de Educação da UNICAMP, 2006.

BRASIL. Ministério da Saúde. Estatuto do idoso. Brasília, 2003.

BRASIL. Ministério da Saúde. Política nacional de saúde da pessoa idosa. Brasília, 2006.

BRASIL. Ministério da Saúde. Secretaria de Vigilância em Saúde. Política nacional de promoção da saúde. Brasília: Secretaria de
Atenção à Saúde, 2006.

CALDAS, C.P. Envelhecimento com dependência: responsabilidades e demandas da família. Cadernos de Saúde Pública, v. 19, n.3, p. 773-781, 2003.

CÂMARA, F. M. Percepção da capacidade funcional de idosos: do incremento da força à força das conexões significativas [Dissertação - Mestrado]. Faculdade de Educação física. Universidade São Judas Tadeu, 2005.

FONSECA, F. B.; RIZZOTTO, M. L. F. Construção de instrumento para avaliação sócio-funcional em idosos. Texto Contexto Enfermagem, v. 17, n.2, p. 365-373, 2008.

GARMAN, K. S.; COHEN, H. J. Functional status and the elderly cancer patient. Critical Reviews in Oncology Hematology, v. 43, 
DIAS, E. G. et al. Caracterização das atividades avançadas. Rev. Ter. Ocup. Univ. São Paulo, v. 22, n. 1, p. 45-51, jan./abr. 2011.

p. 191-208, 2002.

GÓMEZ PORTILLA, A.; MARTÍNEZ DE LECEA, C.; CENDOYA, I.; OLABARRÍA, I.; MARTIIN, E.; MAGRACH, L.; ROMERO, E.; CORTÉS, J.; et al. Prevalencia y tratamiento de la patología oncologica en el anciano. El reto que se avecina. Revista Espanola de Enfermagem Dig., v. 100, n. 11, p. 706-715, 2008.

HO, H. K.; MATSUBAYASHI, K.; WADA, T.; KIMURA, M.; KITA, T.; SAIJOH, K. Factors associated with ADL dependence: A comparative study of residential care home and communitydwelling elderly in Japan. Geriatrics \& Gerontology International, v. 2, p. 80-86, 2002.

JAVIER GÓMEZ, P. El anciano frágil. Detección, prevención e intervención en situaciones de debilidad y deterioro de su salud. Servicio de Promoción de la Salud. Instituto de Salud Pública. Madri: Consejería de sanidad, 2003.

JARDIM, V. C. F. S. Perfil epidemiológico de mulheres idosas participantes de grupos de convivência. [Dissertação - Mestrado] Rio de Janeiro: Centro de Pesquisas Aggeu Magalhães/ Fundação Oswaldo Cruz, 2007.

JOHNSON, R. J.; WOLINSKY, F. D. The structure of health status among older adults: disease, disability,functional limitation, and perceived health. Journal of Health and Social Behavior, v. 34, n. 2, p. 105-121, 1993.

LOBO, A.; PEREIRA, A. Idoso Institucionalizado: funcionalidade e Aptidão Física. Revista Referência II. ${ }^{a}$ Série, n.4, 2007.

LOZANO, G. Terapia ocupacional em los servicios de atención diurna em psicogeriatría. In: DURANTE MOLINA, P.; TARRÉS, P. Terapia ocupacional en geriatría: principios y práctica. $2 \mathrm{a}$. ed. Madrid: Elsevier, 2004. p. 239-251.

LUOH, M. C.; HERZOG, A. R. Individual consequences of volunteer and paid work in old age: Hhealth and mortality. Journal of Health and Social Behavior, v. 43, n. 4, p. 490-509, 2002.

LUBBEN, J. E. Social support networks among elderly Chinese Americans in Los Angeles. In: CHI, I.; CHAPELL, N. L.; LUBBEN, J. E. Elderly Chinese in pacific rim countries: social support and integration. Hong Kong: Hong Kong University Press, 2001.

MANUBENS BERTRAN, J. M. Los trastornos funcionales en lo cotidiano. In: MARTÍNEZ LAGE, J. M.; PASCUAL MILLÁN, L. F. Alzheimer 2003: ¿qué hay de nuevo? Madri: Aula Medica Ediciones, 2003. p: 115-120.

MARTOS MARTÍN, D. A. J. Variables mediadoras en la relación entre el deterioro cognitivo y la capacidad funcional. [Tese doutorado]. Granada: Facultad de Psicologia de la Universidad de Granada, 2005.

MELO, D. M. Fragilidade, desempenho de atividades avançadas de vida diária e saúde percebida em idosos atendidos em ambulatório de geriatria. [Dissertação - Mestrado]. Campinas: Universidade Estadual de Campinas, 2009.
MINCATO, P. C.; FREITAS, C. R. Qualidade de vida dos idosos residentes em instituições asilares da cidade de Caxias do Sul, RS. RBCEH, v. 4, n. 1, p. 127-138, 2007.

PAIER, G. S. Development and testing of an instrument to assess functional status in the elderly. [Tese - doutorado]. Pensilvânia: Universidade da Pensilvânia, 1994.

PAIXÃO, J. R. C. M.; REICHENHEIM, M. E. Uma revisão sobre instrumentos de avaliação do estado funcional do idoso. Cadernos de Saúde Pública, v. 21, n. 1, p. 7-19, 2005.

PASCHOAL, S. M. P. Autonomia e independência. In: PAPALÉO NETTO, M. Gerontologia: a velhice e o envelhecimento em visão globalizada. São Paulo: Atheneu, 2002. p.313-323.

PEÑA-CASANOVA, J.; AGULIAR, M.; BERTRAN-SERRA, I.; SANTACRUZ, P.; HERNÁNDEZ, G.; INSA, R.; PUJOL, A.; SOL, J. M.; BLESA, R. y Grupo NORMACODEM. Normalización de instrumentos cognitivos y funcionales para la evaluación de la demencia. (NORMACODEM): objetivos, contenidos y población. Neurologia, v. 11, p. 61-67, 1997.

PENNING, M. J. Hydra Revisited: Substituting Formal for Self and Informal In-Home Care Among Older Adults With Disabilities. The Gerontologist, v. 42, n. 1, p. 4-16, 2002.

PINCUS, T.; SWEARINGEN, C.; WOLFE, F. Toward a multidimensional Health Assessment Questionnaire (MDHAQ)Assessment of Advanced Activities of Daily Living and Psychological Status in the Patient-Friendly Health Assessment Questionnaire Format. Arthritis \& Rheumatism, v. 42, n. 10, p. 2220-2230, 1999.

POLÔNIO-LÓPEZ, B. El processo de terapia ocupacional em geriatria. In:TERAPIA ocupacional em geriatria: 15 casos practicos. Madrid: Editorial Médica Panamericana, 2002. p. 49-66.

POVEDA, R.; BELDA, J.; BARBERÀ, R.; CORT, J. M.; PRAT, J. M.; MATEY, F.; SOLER, C.; DEJOZ, R. Facilitación de la vida cotidiana mediante el diseño y la selección adecuada de tecnologias de apoyo para personas mayores con dependencia funcional. Rev Mult Gerontol, v. 15, n. 3, p. 155-164, 2005.

REUBEN, D. B.; SOLOMON, D. H. Assessment in geriatrics: of caveats and names (editorial). Journal American Geriatric Society, v. 37, p. 570-572, 1989.

REUBEN, D. B.; LALIBERTE, L.; HIRIS, J.; MOR, V. A hierarquical exercise scale to measure function at the advanced activities of daily living (AADL) level. Journal American Geriatric Society, v. 38, p. 855-861, 1990.

RIVERA-ZETINA, D. V.; ESTRADA-HERNÁNDEZ, M. R.; ESTRADA-HERNÁNDEZ, L. O. Posibilidades de la rehabilitación neuropsicológica en población geriátrica con deterioro Cognoscitivo. Med Int Mexico, v. 23, p. 133-137, 2007.

ROLIM, F. L.; FORTI, V. A. M. Atividade fisica e os domínios 
DIAS, E. G. et al. Caracterização das atividades avançadas. Rev. Ter. Ocup. Univ. São Paulo, v. 22, n. 1, p. 45-51, jan./abr. 2011.

da qualidade de vida e do autoconceito no processo de envelhecimento. [Dissertação - Mestrado]. Campinas: Faculdade de Educação física da UNICAMP, 2005.

ROMERO-AYUSO, M.; MORUNO-MIRALLES, P. Terapia ocupacional: teoría y técnicas. Madrid: Elsevier; 2003. p. 237245: Valoración funcional.

ROZZINI, R.; BERTOZZI, B.; BARBISONI, P. Functional status after cardiac surgery in elderly people. Age and Ageing, v. 25, p. 413-415, 1996.

RUBENSTEIN, L. V.; CALKINS, D. R.; GREENFIELD, S.; JETTE, A. M.; MEENAN, R. F.; NEVINS, M. A.; RUBENSTEIN, L. Z.; WASSON, J. H.; WILLIAMS, M. E. Health status assessment for elderly patients. $J A G S$, v. 37, p. 562-569, 1988.

SALVADORES, P. Enfermería y rehabilitación. Um proyecto de futuro. In: MIANGOLARRA PAGE, J. C.; ALGUACIL DIEGO,
I. M.; ÁGUILAR MATURANA, A. M. Rehabilitacion clinica integral funcionamiento y discapacidad. Madrid: Elsevier, 2003. p. 102-109.

SANJOAQUÍN ROMERO, A. C.; FERNÁNDEZ ARÍN, E.; MESA LAMPRÉ, M. P.; GARCÍA-ARILLA CALVO, E. Valoración geriátrica Integral. In: Sociedad Española Geriatría y Gerontología (SEGG). Tratado de geriatría para residentes. Pamplona, 2006. p. 59-68.

SILVA, A. C. Atividade fisica habitual e saúde multidimensional de idosos na cidade de Goiânia-GO. [Dissertação - Mestrado]. Blumenau: Faculdade de Educação Física da Universidade Federal de Santa Catarina, 2005.

SINDACO, D. D.; ZUCCALÀ, G.; PULIGNANO, G.; COCCHI, A. La valutazione multidimensionale dell'anziano con scompenso cardiac. Italian Heart Journal, v. 5, n. 10, p. 26-36, 2004.

Recebido para publicação: 01/12/10

Aceito para publicação: 10/05/11 